\title{
A QUADRATURE FORMULA WITH ZEROS OF BESSEL FUNCTIONS AS NODES
}

\author{
GEORGI R. GROZEV AND QAZI I. RAHMAN
}

\begin{abstract}
A quadrature formula for entire functions of exponential type wherein the nodes are the zeros of the Bessel function of the first kind was recently obtained by C. Frappier and P. Olivier. Here the condition imposed on the function is relaxed. Some applications of the formula are also given.
\end{abstract}

\section{INTRODUCTION AND STATEMENT OF RESULTS}

If $f$ is an entire function of exponential type $2 \tau>0$ belonging to $L^{1}(-\infty, \infty)$ then $([3])$

$$
\int_{-\infty}^{\infty} f(x) d x=\frac{\pi}{\tau} \sum_{k=-\infty}^{\infty} f\left(\frac{(2 k-1) \pi}{2 \tau}\right),
$$

where the series is absolutely convergent.

If $f$ belongs to $L^{1}(-\infty, \infty)$, then so do the functions $(f(x)+f(-x)) / 2$ and $(f(x)-f(-x)) / 2$. Since $(f(x)-f(-x)) / 2$ is odd, $\int_{-\infty}^{\infty} f(x) d x$ is nothing but $\int_{0}^{\infty}(f(x)+f(-x)) d x$. Hence (1) may be written as

$$
\left(1^{\prime}\right) \int_{0}^{\infty}(f(x)+f(-x)) d x=\frac{\pi}{\tau} \sum_{k=1}^{\infty}\left(f\left(\frac{(2 k-1) \pi}{2 \tau}\right)+f\left(-\frac{(2 k-1) \pi}{2 \tau}\right)\right) .
$$

Applying (1) to the function $f(z)+f(-z)$, we see that in this form, the formula is valid even if $f(x)+f(-x)$ belongs to $L^{1}[0, \infty)$ without $f$ being in $L^{1}(-\infty, \infty)$.

Let $J_{\alpha}(z)$ be the Bessel function of the first kind of order $\alpha$. We shall denote by $j_{1}(\alpha), j_{2}(\alpha), \ldots, j_{k}(\alpha), \ldots$ the zeros of $\frac{J_{\alpha}(z)}{z^{\alpha}}$ in the right half-plane arranged in ascending order of magnitude.

Recently, Frappier and Olivier [4] have proved the following result which we find very interesting.

Received by the editor March 4, 1994.

1991 Mathematics Subject Classification. Primary 65D32, 41A55 , 30D10, 33C10.

Key words and phrases. Quadrature formulae, entire functions of exponential type, zeros of Bessel functions, Carlson's theorem.

The first author gratefully acknowledges the support from the Natural Sciences and Engineering Research Council of Canada Grant No. A-3081 (Q.I.Rahman); he was also partially supported by Grant No.MM-15 from the Bulgarian Ministry of Sciences. 
Theorem A. If $\mathfrak{R} e \alpha>-1$, then the quadrature formula

$$
\begin{aligned}
& \int_{0}^{\infty} \cdot x^{2 \alpha+1}(f(x)+f(-x)) d x \\
&=\frac{2}{\tau^{2 \alpha+2}} \sum_{k=1}^{\infty} \frac{\left(j_{k}(\alpha)\right)^{2 \alpha}}{\left(J_{\alpha}^{\prime}\left(j_{k}(\alpha)\right)\right)^{2}}\left(f\left(\frac{j_{k}(\alpha)}{\tau}\right)+f\left(-\frac{j_{k}(\alpha)}{\tau}\right)\right)
\end{aligned}
$$

holds for every entire function $f$ of exponential type $2 \tau$ such that $f(x)=$ $O\left(|x|^{-\delta}\right), \quad x \rightarrow \pm \infty$ with $\delta>2 \Re e \alpha+2$.

Formula (2) reduces to ( $\left.1^{\prime}\right)$ when $\alpha=-\frac{1}{2}$ since

$$
\frac{J_{-\frac{1}{2}}(\tau z)}{(\tau z)^{-\frac{1}{2}}}=\sqrt{\frac{2}{\pi}} \cos (\tau z) .
$$

However, in the case $\alpha=-\frac{1}{2}$, formula (2) holds for every entire function $f$ of exponential type $2 \tau$ such that $x^{2 \alpha+1}(f(x)+f(-x))$ belongs to $L^{1}[0, \infty)$. It is therefore natural to wonder if the same can be said for other values of $\alpha$ too. We shall show that this is indeed the case if $\alpha$ is real and $>-1$. To be precise, we have

Theorem 1. If $\alpha>-1$, then (2) holds for every entire function $f$ of exponential type $2 \tau$ such that $x^{2 \alpha+1}(f(x)+f(-x))$ belongs to $L^{1}[0, \infty)$. Besides, the series on the right-hand side of (2) is absolutely convergent.

It may be mentioned that the zeros $j_{1}(\alpha), j_{2}(\alpha), \ldots, j_{k}(\alpha), \ldots$ are all real when $\alpha$ is real and $>-1$.

If $f$ is integrable on $[1, X]$ for all $X>1$ and

$$
\lim _{X \rightarrow \infty} \int_{1}^{X} f(x) d x
$$

exists, then we denote the limit by $\int_{1}^{\rightarrow \infty} f(x) d x$ and say that $f$ is integrable in the sense of Cauchy on $[1, \infty)$. If $f$ is integrable on $[\xi, 1]$ for all $\xi \in(0,1)$ and

$$
\lim _{\xi \rightarrow 0} \int_{\xi}^{1} f(x) d x
$$

exists, then we denote the limit by $\int_{\rightarrow_{0}}^{1} f(x) d x$ and say that $f$ is integrable in the sense of Cauchy on $(0,1]$. If $\vec{f}$ is integrable in the sense of Cauchy on $[1, \infty)$ as well as on $(0,1]$, then we say that $f$ is integrable (in the sense of Cauchy) on $(0, \infty)$ and denote the integral by

$$
\int_{\rightarrow 0}^{\rightarrow \infty} f(x) d x .
$$

The question arises if in Theorem 1 it would be enough to assume the function $x^{2 \alpha+1}(f(x)+f(-x))$ to be integrable in the sense of Cauchy on $(0, \infty)$. The answer is no. Indeed, the function

$$
f_{*}(z)=\frac{J_{\alpha}(\tau z) J_{\alpha+1}(\tau z)}{z^{2 \alpha+1}}
$$

which was considered in $[4, \S 5.2]$ serves as a counterexample. This function is of exponential type $2 \tau$ and is integrable in the sense of Cauchy on $(0, \infty)$; the value of the integral is $\frac{1}{\tau}[8, p .406]$ whereas the right-hand side of $(2)$ is 0 . However, we shall prove the following 
Theorem 2. Let $\alpha>-1$ and $f$ be an entire function of exponential type $\sigma<2 \tau$ such that $x^{2 \alpha+1}(f(x)+f(-x))$ is integrable in the sense of Cauchy on $(0, \infty)$. Then

$$
\begin{aligned}
\int_{\rightarrow 0}^{\rightarrow \infty} x^{2 \alpha+1} & (f(x)+f(-x)) d x \\
= & \frac{2}{\tau^{2 \alpha+2}} \sum_{k=1}^{\infty} \frac{\left(j_{k}(\alpha)\right)^{2 \alpha}}{\left(J_{\alpha}^{\prime}\left(j_{k}(\alpha)\right)\right)^{2}}\left(f\left(\frac{j_{k}(\alpha)}{\tau}\right)+f\left(-\frac{j_{k}(\alpha)}{\tau}\right)\right),
\end{aligned}
$$

if the series on the right is convergent.

The following result was also proved in [4] to which we refer the reader for the definition of the nodal function $\omega$.

Theorem B. Let $\alpha>-1$. Among all quadrature formulae of the form

$$
\int_{0}^{\infty} x^{2 \alpha+1}(f(x)+f(-x)) d x=\sum_{k=-\infty}^{\infty} \lambda_{k} f\left(x_{k}\right)
$$

having $\omega$ as nodal function, only one is valid for all entire functions of exponential type $2 \tau$ satisfying $f(x)=O\left(|x|^{-\delta}\right), x \rightarrow \pm \infty, \delta>2 \alpha+2$. This formula is (2) and the associated nodal function is

$$
\omega_{e}(x):=\frac{\left(j_{m}(\alpha)\right)^{\alpha}}{J_{\alpha}^{\prime}\left(j_{m}(\alpha)\right)} \frac{J_{\alpha}(\tau x)}{x^{\alpha}\left(\tau x-j_{m}(\alpha)\right)} .
$$

Moreover, $\omega_{e}$ minimizes the integral

$$
\int_{0}^{\infty} x^{2 \alpha+1}\left(\omega^{2}(x)+\omega^{2}(-x)\right) d x
$$

over all nodal functions $\omega$.

The proof of Theorem B as given in [4] allows us, in view of Theorem 1, to state the following

Theorem 3. Theorem B holds if instead of " $f(x)=O\left(|x|^{-\delta}\right), x \rightarrow \pm \infty, \delta>$ $2 \alpha+2$ " we assume that $x^{2 \alpha+1}(f(x)+f(-x))$ belongs to $L^{1}[0, \infty)$.

As an application of Theorem 1 we prove

Theorem 4. If $f$ is an entire function of exponential type $\tau$ such that

$$
|x|^{\alpha+\frac{1}{2}} f(x) \in L^{2}(\mathbb{R})
$$

for some $\alpha>-1$, then

$$
\int_{-\infty}^{\infty}|x|^{2 \alpha+1}|f(x)|^{2} d x=\frac{2}{\tau^{2 \alpha+2}} \sum_{\substack{k=-\infty \\ k \neq 0}}^{\infty} \frac{\left|j_{k}(\alpha)\right|^{2 \alpha}}{\left(J_{\alpha}^{\prime}\left(\left|j_{k}(\alpha)\right|\right)\right)^{2}}\left|f\left(\frac{j_{k}(\alpha)}{\tau}\right)\right|^{2},
$$

where $j_{-k}(\alpha)=-j_{k}(\alpha)$ for all $k \neq 0$.

In the case $\alpha=-\frac{1}{2},(5)$ reduces to the following well-known formula of Plancherel and Pólya ([7, p.116, formula (52)])

$$
\int_{-\infty}^{\infty}|f(x)|^{2} d x=\frac{\pi}{\tau} \sum_{k=-\infty}^{\infty}\left|f\left(\frac{(2 k-1) \pi}{2 \tau}\right)\right|^{2} .
$$

Here is an obvious corollary of Theorem 4 . 
Corollary 1. If $f$ is an entire function of exponential type $\pi$ such that (4) holds for some $\alpha>-1$ and $f$ vanishes at all the points

$$
\frac{j_{k}(\alpha)}{\pi}, k= \pm 1, \pm 2, \pm 3, \ldots,
$$

then $f$ is identically zero.

This result is to be compared with a classical theorem of F. Carlson according to which, if $f$ is an entire function of exponential type $<\pi$ and vanishes at the points $n=0, \pm 1, \pm 2, \ldots$, then it is identically zero. We refer the reader to [2, Chapter 9] for various extentions of Carlson's theorem. It may be noted that in the above corollary the function is allowed to be of order 1 type $\pi$ as long as (4) holds.

Remark 1. The example $\frac{J_{\alpha}(\pi z)}{(\pi z)^{\alpha}}$ shows that the conclusion of the above corollary may not hold if (4) is not satisfied.

Remark 2. If a function is of exponential type $\pi$ satisfying (4), then it may not be identically zero unless it vanishes at all the points $\frac{j_{k}(\alpha)}{\pi}, k= \pm 1, \pm 2, \ldots$. This is shown by the example

$$
f(z):=\frac{J_{\alpha}(\pi z)}{(\pi z)^{\alpha}\left(\pi z-j_{n}(\alpha)\right)},
$$

where $n \in\{ \pm 1, \pm 2, \pm 3, \ldots\}$.

As a special case of Theorem 4 we have

Corollary 2. If $f$ satisfies the conditions of Theorem 4, then

$$
\left|f\left(\frac{j_{n}(\alpha)}{\tau}\right)\right| \leq \frac{\tau^{\alpha+1}}{\sqrt{2}} \frac{\left|J_{\alpha}^{\prime}\left(j_{n}(\alpha)\right)\right|}{\left|j_{n}(\alpha)\right|^{\alpha}}\left(\int_{-\infty}^{\infty}|x|^{2 \alpha+1}|f(x)|^{2} d x\right)^{1 / 2} \quad(n=1,2, \ldots) .
$$

The inequality is sharp; indeed, it becomes an equality for the function defined in (6).

\section{AUXILIARY RESULTS}

Let $\phi(t)$ be a nonnegative, nondecreasing, and convex (nonconcave) function of $\log t$, with $\phi(0+)=0$ but $\phi(t)$ not identically zero. Further, let $\phi\left(e^{-t}\right)=$ $O\left(t^{-1 / 2}\right)$ as $t \rightarrow+\infty$. It was proved by Boas [1, Theorem 2] that if $f$ is regular and of exponential type $c$ in the closed right half-plane such that for some $\mu>0, \lambda>2$,

$$
\int_{0}^{\infty}\left\{\phi\left(|f(x)|^{\mu}\right)\right\}^{\lambda} d x<\infty
$$

then for an arbitrary increasing sequence of positive numbers $\lambda_{0}, \lambda_{1}, \lambda_{2}, \ldots$ with $\lambda_{k+1}-\lambda_{k} \geq 2 \delta>0$ we have

$$
\sum_{k=0}^{\infty}\left\{\phi\left(e^{-c^{\prime} \mu \delta}\left|f\left(\lambda_{k}\right)\right|^{\mu}\right\}^{\lambda}<\infty,\right.
$$

if $c^{\prime}>c$. Going through the proof of this result, the reader will notice an obvious misprint in [1, Lemma 4] concerning the assumption “ $\phi\left(e^{-t}\right)=O\left(t^{-1 / 2}\right)$ as $t \rightarrow+\infty$ ". The conditions imposed on $\phi$ are clearly satisfied by $\phi(t)=t$. 
Given a sequence $\lambda_{1}, \lambda_{2}, \lambda_{3}, \ldots$ such that $\lambda_{1}>0, \lambda_{k+1}-\lambda_{k} \geq 2 \delta>0$, and a function $f$ regular and of exponential type in the open right half-plane we may apply the above result of Boas to the function $f\left(z+\frac{1}{2} \lambda_{1}\right)$ taking $\phi(t)=t, \mu=\frac{1}{3}, \lambda=3$ to obtain

Lemma 1. Let $\left\{\lambda_{k}\right\}$ be an increasing sequence of positive numbers with $\lambda_{k+1}-$ $\lambda_{k} \geq 2 \delta>0$. If $f$ is regular and of exponential type in the open right half-plane such that

$$
\int_{0}^{\infty}|f(x)| d x<\infty
$$

then

$$
\sum_{k=1}^{\infty}\left|f\left(\lambda_{k}\right)\right|<\infty
$$

Hereafter, let

$$
\varphi_{h}(z):=\left(\frac{\sin h z}{h z}\right)^{2}
$$

where $h>0$.

Lemma 2. We have

$$
\int_{-\infty}^{\infty}\left|\varphi_{h}^{\prime}(x)\right| d x \leq 2 \pi .
$$

Besides, for $\alpha>-1$ and all $\gamma>0$

$$
\sum_{k=1}^{\infty}\left|\varphi_{h}\left(\gamma j_{k+1}(\alpha)\right)-\varphi_{h}\left(\gamma j_{k}(\alpha)\right)\right| \leq \pi
$$

Proof. Since $\varphi_{h}$ is an entire function of exponential type $2 h$ belonging to $L^{1}(-\infty, \infty)$, we have [2, Theorem 11.3.1]

$$
\int_{-\infty}^{\infty}\left|\varphi_{h}^{\prime}(x)\right| d x \leq 2 h \int_{-\infty}^{\infty}\left|\varphi_{h}(x)\right| d x=2 \pi .
$$

Further, using the fundamental theorem of integral calculus, we have

$$
\begin{aligned}
\sum_{k=1}^{\infty}\left|\varphi_{h}\left(\gamma j_{k+1}(\alpha)\right)-\varphi_{h}\left(\gamma j_{k}(\alpha)\right)\right| & =\gamma \sum_{k=1}^{\infty}\left|\int_{j_{k}(\alpha)}^{j_{k+1}(\alpha)} \varphi_{h}^{\prime}(\gamma x) d x\right| \\
& \leq \frac{1}{2} \int_{-\infty}^{\infty}\left|\varphi_{h}^{\prime}(x)\right| d x \leq \pi .
\end{aligned}
$$

Lemma 3 [5, §2.2]. Let $f$ be regular and of exponential type in the open right half-plane. If $f$ is integrable in the sense of Cauchy on $[1, \infty)$, then $f(x) \rightarrow 0$ as $x \rightarrow \infty$, and so $f(x)$ is bounded for $x \geq 1$.

Lemma 4 [5, Lemma 1]. If $f$ is integrable in the sense of Cauchy on $[1, \infty)$, then $\int_{1}^{\rightarrow \infty} \varphi_{h}(x) f(x) d x$ exists for all $h>0$ and

$$
\lim _{h \rightarrow 0} \int_{1}^{\rightarrow \infty} \varphi_{h}(x) f(x) d x=\int_{1}^{\rightarrow \infty} f(x) d x
$$

provided $f$ is bounded on $[1, \infty)$. 
Lemma $4^{\prime}$. If $f$ is integrable in the sense of Cauchy on $(0,1]$, then

$$
\int_{\rightarrow 0}^{1} \varphi_{h}(x) f(x) d x
$$

exists for all $h>0$ and

$$
\lim _{h \rightarrow 0} \int_{\rightarrow 0}^{1} \varphi_{h}(x) f(x) d x=\int_{\rightarrow 0}^{1} f(x) d x .
$$

Proof. For every $\varepsilon>0$, there exists $X_{\varepsilon}>0$ such that

$$
\left|\int_{x_{1}}^{x_{2}} f(x) d x\right|<\varepsilon \quad \text { for } 0<x_{1}<x_{2} \leq X_{\varepsilon} .
$$

Hence, if $F(x):=\int_{x}^{X_{\varepsilon}} f(t) d t \quad\left(0<x \leq X_{\varepsilon}\right)$, then

$$
|F(x)|<\varepsilon \quad \text { for } 0<x \leq X_{\varepsilon} .
$$

Let $0<x_{1}<x_{2} \leq X_{\varepsilon}$. Integration by parts gives

$$
\int_{x_{1}}^{x_{2}} \varphi_{h}(x) f(x) d x=-\varphi_{h}\left(x_{2}\right) F\left(x_{2}\right)+\varphi_{h}\left(x_{1}\right) F\left(x_{1}\right)+\int_{x_{1}}^{x_{2}} \varphi_{h}^{\prime}(x) F(x) d x,
$$

which by virtue of (7) and (9) implies

$$
\left|\int_{x_{1}}^{x_{2}} \varphi_{h}(x) f(x) d x\right| \leq 2(1+\pi) \varepsilon
$$

i.e., $\int_{\rightarrow 0}^{1} \varphi_{h}(x) f(x) d x$ exists for all $h>0$.

Note that (10) holds for all $h$ and so, using (9), we obtain

$$
\left|\int_{\rightarrow 0}^{X_{\varepsilon}} \varphi_{h}(x) f(x) d x-\int_{\rightarrow 0}^{X_{\varepsilon}} f(x) d x\right| \leq(3+2 \pi) \varepsilon .
$$

Since

$$
\left|\int_{X_{\varepsilon}}^{1} \varphi_{h}(x) f(x) d x-\int_{X_{\varepsilon}}^{1} f(x) d x\right|<\varepsilon
$$

for all sufficiently small $h$, we conclude that (8) holds.

Remark 3. The above proof differs only in some details from that of Lemma 4 as presented in [5].

Lemma 5. If $f$ satisfies the conditions of Theorem 1, then the series on the right-hand side of (2) is absolutely convergent.

Proof. Let

$$
\lambda_{k}:=\frac{1}{\tau} j_{k}(\alpha) \quad(k=1,2, \ldots) .
$$

Since $j_{1}(\alpha), j_{2}(\alpha), \ldots, j_{k}(\alpha), \ldots$, are all simple, positive and (see [8, p. 506])

$$
\begin{aligned}
j_{k}(\alpha)= & \left(k+\frac{1}{2} \alpha-\frac{1}{4}\right) \pi-\frac{4 \alpha^{2}-1}{8 \pi\left(k+\frac{1}{2} \alpha-\frac{1}{4}\right)} \\
& -\frac{\left(4 \alpha^{2}-1\right)\left(28 \alpha^{2}-31\right)}{384 \pi^{3}\left(k+\frac{1}{2} \alpha-\frac{1}{4}\right)^{3}}-\cdots,
\end{aligned}
$$

there exists a positive number $\delta$ such that $\lambda_{k+1}-\lambda_{k} \geq 2 \delta$. 
According to hypothesis we have

$$
\int_{0}^{\infty}|x|^{2 \alpha+1}|f(x)+f(-x)| d x<\infty
$$

and so by Lemma 1

$$
\begin{aligned}
\sum_{k=1}^{\infty}\left(\frac{1}{\tau} j_{k}(\alpha)\right)^{2 \alpha+1} & \left|f\left(\frac{j_{k}(\alpha)}{\tau}\right)+f\left(-\frac{j_{k}(\alpha)}{\tau}\right)\right| \\
& =\sum_{k=1}^{\infty}\left|\lambda_{k}\right|^{2 \alpha+1}\left|f\left(\lambda_{k}\right)+f\left(-\lambda_{k}\right)\right|<\infty .
\end{aligned}
$$

Now we recall the formula ( $[9$, p. 368], [6, p. 198 (Lemma 14)])

$$
J_{\alpha}^{\prime}(z)=-\left(\frac{2}{\pi z}\right)^{1 / 2} \sin \left(z-\frac{\alpha \pi}{2}-\frac{\pi}{4}\right)+O\left(\frac{1}{|z|^{3 / 2}}\right), z \in \mathbb{R}, \quad z \rightarrow \infty .
$$

It follows from (11) that for all large $k$

$$
\left|\sin \left(j_{k}(\alpha)-\frac{\alpha \pi}{2}-\frac{\pi}{4}\right)\right|>\frac{1}{\sqrt{2}} .
$$

So, if $k$ is sufficiently large (say $k \geq K_{1}$ ), then

$$
\begin{aligned}
\left|J_{\alpha}^{\prime}\left(j_{k}(\alpha)\right)\right| & \geq\left(\frac{1}{\pi j_{k}(\alpha)}\right)^{1 / 2}-O\left(\left(\frac{1}{j_{k}(\alpha)}\right)^{3 / 2}\right) \\
& >\frac{1}{\sqrt{2} \sqrt{\pi j_{k}(\alpha)}},
\end{aligned}
$$

and

$$
\begin{aligned}
\frac{\left(j_{k}(\alpha)\right)^{2 \alpha}}{\left(J_{\alpha}^{\prime}\left(j_{k}(\alpha)\right)\right)^{2}} \mid f\left(\frac{j_{k}(\alpha)}{\tau}\right) & +f\left(-\frac{j_{k}(\alpha)}{\tau}\right) \mid \\
& <2 \pi\left(j_{k}(\alpha)\right)^{2 \alpha+1}\left|f\left(\frac{j_{k}(\alpha)}{\tau}\right)+f\left(-\frac{j_{k}(\alpha)}{\tau}\right)\right|
\end{aligned}
$$

Besides, $J_{\alpha}^{\prime}\left(j_{k}(\alpha)\right) \neq 0$ for all $k$ since the positive zeros of $J_{\alpha}$ are all simple. Hence, in view of (12), the lemma holds.

\section{Proofs of THE THEOREMS}

Proof of Theorem 1. Without loss of generality we may assume $\tau=\pi$. Then

$$
\psi_{\varepsilon}(z):=\varphi_{\varepsilon}(z) \cdot(f(z)+f(-z)) \quad(\varepsilon>0)
$$

is an even entire function of exponential type $2(\pi+\varepsilon)$.

The function $z^{2 \alpha+1}(f(z)+f(-z))$ is regular and of exponential type in Re $z>0$. Besides, according to our assumption, it belongs to $L^{1}[0, \infty)$ and so by Lemma 3

$$
|x|^{2 \alpha+1}|f(x)+f(-x)| \rightarrow 0 \quad \text { as } x \rightarrow \infty
$$


Hence,

$$
|f(x)+f(-x)|=O\left(|x|^{-(2 \alpha+1)}\right) \quad \text { as } x \rightarrow \pm \infty,
$$

which implies that

$$
\left|\psi_{\varepsilon}(x)\right|=O\left(|x|^{-(2 \alpha+3)}\right) \quad \text { as } x \rightarrow \pm \infty .
$$

Thus the function $\psi_{\varepsilon}(z)$ satisfies the conditions of Theorem A with $2(\pi+\varepsilon)$ instead of $2 \tau$. We therefore have

$$
\int_{0}^{\infty} x^{2 \alpha+1}\left(2 \psi_{\varepsilon}(x)\right) d x=\frac{2}{(\pi+\varepsilon)^{2 \alpha+2}} \sum_{k=1}^{\infty} \frac{\left(j_{k}(\alpha)\right)^{2 \alpha}}{\left(J_{\alpha}^{\prime}\left(j_{k}(\alpha)\right)\right)^{2}} \cdot 2 \psi_{\varepsilon}\left(\frac{j_{k}(\alpha)}{\pi+\varepsilon}\right) .
$$

Next we show that for each $\delta>0$ there exists $K_{0}$ such that

$$
\frac{2}{(\pi+\varepsilon)^{2 \alpha+2}} \sum_{k=K_{0}}^{\infty}\left|\frac{\left(j_{k}(\alpha)\right)^{2 \alpha}}{\left(J_{\alpha}^{\prime}\left(j_{k}(\alpha)\right)\right)^{2}} \psi_{\varepsilon}\left(\frac{j_{k}(\alpha)}{\pi+\varepsilon}\right)\right|<\delta
$$

if $\varepsilon \in\left[0, \frac{1}{2}\right]$. In view of (11) we can choose an integer $K_{2}$ such that for $\varepsilon \in\left[0, \frac{1}{2}\right]$,

$$
\frac{1}{\pi+\varepsilon}\left(j_{k+1}(\alpha)-j_{k}(\alpha)\right)>\frac{1}{2} \quad \text { for } k \geq K_{2} \text {. }
$$

Let $F(x):=x^{2 \alpha+1}(f(x)+f(-x))$ and $\xi_{k}(1 \leq k<\infty)$ be the smallest number in $\left[k-\frac{1}{2}, k+\frac{1}{2}\right]$ such that

$$
\left|F\left(\xi_{k}\right)\right|=\max _{k-\frac{1}{2} \leq x \leq k+\frac{1}{2}}|F(x)|
$$

The points $\xi_{k}$ which lie in $\left[k-\frac{1}{2}, k\right)$ form a subsequence $\left\{\xi_{n}^{\prime}\right\}$ and those which lie in $\left[k, k+\frac{1}{2}\right]$ form another subsequence $\left\{\xi_{n}^{\prime \prime}\right\}$. Obviously $\xi_{n+1}^{\prime}-\xi_{n}^{\prime} \geq \frac{1}{2}$ and $\xi_{n+1}^{\prime \prime}-\xi_{n}^{\prime \prime} \geq \frac{1}{2}$ and so by Lemma 1

$$
\sum_{n=1}^{\infty}\left|F\left(\xi_{n}^{\prime}\right)\right|<\infty, \sum_{n=1}^{\infty}\left|F\left(\xi_{n}^{\prime \prime}\right)\right|<\infty \text {. }
$$

As such,

$$
\sum_{k=1}^{\infty}\left|F\left(\xi_{k}\right)\right|<\infty
$$

Therefore there exists $K_{0}>\max \left\{K_{1}, K_{2}\right\}$ such that

By (13),

$$
8 \sum_{k=\left[\frac{6}{9} K_{0}\right]}^{\infty}\left|F\left(\xi_{k}\right)\right|<\delta
$$

$$
\begin{aligned}
\left|\frac{2}{(\pi+\varepsilon)^{2 \alpha+2}} \sum_{k=K_{0}}^{\infty} \frac{\left(j_{k}(\alpha)\right)^{2 \alpha}}{\left(J_{\alpha}^{\prime}\left(j_{k}(\alpha)\right)\right)^{2}} \cdot \psi_{\varepsilon}\left(\frac{j_{k}(\alpha)}{\pi+\varepsilon}\right)\right| \\
\quad \leq \frac{4 \pi}{(\pi+\varepsilon)} \sum_{k=K_{0}}^{\infty}\left|\frac{j_{k}(\alpha)}{\pi+\varepsilon}\right|^{2 \alpha+1}\left|f\left(\frac{j_{k}(\alpha)}{\pi+\varepsilon}\right)+f\left(-\frac{j_{k}(\alpha)}{\pi+\varepsilon}\right)\right| \\
\quad=\frac{4 \pi}{\pi+\varepsilon} \sum_{k=K_{0}}^{\infty}\left|F\left(\frac{j_{k}(\alpha)}{\pi+\varepsilon}\right)\right|
\end{aligned}
$$


Because of (16), intervals of the form $\left[k-\frac{1}{2}, k+\frac{1}{2}\right]$ can contain at most two points of the sequence $\left\{\frac{j_{k}(\alpha)}{\pi+\varepsilon}\right\}_{k \geq K_{2}}$. Hence,

$$
\frac{4 \pi}{\pi+\varepsilon} \sum_{k=K_{0}}^{\infty}\left|F\left(\frac{j_{k}(\alpha)}{\pi+\varepsilon}\right)\right| \leq 4 \times 2 \sum_{k=\left[\frac{6}{9} K_{0}\right]}^{\infty}\left|F\left(\xi_{k}\right)\right|<\delta,
$$

i.e., (15) holds.

It is clear that as $\varepsilon \rightarrow 0$ the function

$$
\frac{1}{(\pi+\varepsilon)^{2 \alpha+2}} \psi_{\varepsilon}(z) \text { tends to } \frac{1}{\pi^{2 \alpha+2}}(f(z)+f(-z))
$$

uniformly on all compact subsets of $\mathbb{C}$. Hence, for any $\delta>0$ and any $K \in \mathbb{N}$ there exists a positive $\varepsilon_{0}$ depending on $\delta$ and $K$ such that

$$
\begin{aligned}
\mid \frac{2}{\pi^{2 \alpha+2}} \sum_{k=1}^{K} \frac{\left(j_{k}(\alpha)\right)^{2 \alpha}}{\left(J_{\alpha}^{\prime}\left(j_{k}(\alpha)\right)\right)^{2}} & \left(f\left(\frac{j_{k}(\alpha)}{\pi}\right)+f\left(-\frac{j_{k}(\alpha)}{\pi}\right)\right) \\
- & \frac{2}{(\pi+\varepsilon)^{2 \alpha+2}} \sum_{k=1}^{K} \frac{\left(j_{k}(\alpha)\right)^{2 \alpha}}{\left(J_{\alpha}^{\prime}\left(j_{k}(\alpha)\right)\right)^{2}} \psi_{\varepsilon}\left(\frac{j_{k}(\alpha)}{\pi+\varepsilon}\right) \mid<\delta
\end{aligned}
$$

if $\varepsilon \in\left[0, \varepsilon_{0}\right]$.

Now let

$$
I(f):=\int_{0}^{\infty} x^{2 \alpha+1}(f(x)+f(-x)) d x
$$

and

$$
Q_{\tau}(f):=\frac{2}{\tau^{2 \alpha+2}} \sum_{k=1}^{\infty} \frac{\left(j_{k}(\alpha)\right)^{2 \alpha}}{\left(J_{\alpha}^{\prime}\left(j_{k}(\alpha)\right)\right)^{2}}\left(f\left(\frac{j_{k}(\alpha)}{\tau}\right)+f\left(-\frac{j_{k}(\alpha)}{\tau}\right)\right)
$$

Then

$$
\begin{aligned}
\left|I(f)-Q_{\pi}(f)\right| & =\left|I(f)+I\left(\psi_{\varepsilon}\right)-I\left(\psi_{\varepsilon}\right)-Q_{\pi}(f)\right| \\
& \leq\left|I(f)-I\left(\psi_{\varepsilon}\right)\right|+\left|Q_{\pi}(f)-I\left(\psi_{\varepsilon}\right)\right| \\
& =\left|I(f)-I\left(\psi_{\varepsilon}\right)\right|+\left|Q_{\pi}(f)-Q_{\pi+\varepsilon}\left(\psi_{\varepsilon}\right)\right|,
\end{aligned}
$$

where in the last step we have used (14). By Lemmas 3, 4 and $4^{\prime}$, for each $\delta>0$ there exists $\varepsilon_{1}>0$ such that

$$
\left|I(f)-I\left(\psi_{\varepsilon}\right)\right|<\delta \quad \text { for } 0<\varepsilon<\varepsilon_{1} .
$$

Further, by (15), (17) and Lemma 5 we conclude that

$$
\left|Q_{\pi}(f)-Q_{\pi+\varepsilon}\left(\psi_{\varepsilon}\right)\right|<3 \delta
$$

for all $\varepsilon \in\left(0, \min \left\{\varepsilon_{0}, \frac{1}{2}\right\}\right)$. Hence, for $\varepsilon \in\left(0, \min \left\{\varepsilon_{0}, \frac{1}{2}, \varepsilon_{1}\right\}\right)$ we have

$$
\left|I(f)-Q_{\pi}(f)\right|<4 \delta .
$$

Since $\delta$ is an arbitrary positive number, we must have $I(f)=Q_{\pi}(f)$. This completes the proof of Theorem 1, since the absolute convergence of the series on the right-hand side of (2) has already been proved in Lemma 5.

Proof of Theorem 2. The function

$$
\psi_{\varepsilon}(z):=\varphi_{\varepsilon}(z)(f(z)+f(-z))
$$


satisfies the conditions of Theorem 1 for all $\varepsilon \in\left(0, \tau-\frac{\sigma}{2}\right)$ and therefore $I\left(\psi_{\varepsilon}\right)=$ $Q_{\tau}\left(\psi_{\varepsilon}\right)$. From Lemmas 3,4 and $4^{\prime}$ it follows that

$$
\int_{\rightarrow 0}^{\rightarrow \infty} x^{2 \alpha+1} \psi_{\varepsilon}(x) d x \rightarrow \int_{\rightarrow 0}^{\rightarrow \infty} x^{2 \alpha+1}(f(x)+f(-x)) d x \quad \text { as } \varepsilon \rightarrow 0 .
$$

Let $\delta$ be an arbitrary positive number and choose the smallest $N_{\delta}$ such that

$$
\left|\sum_{k=N_{\delta}}^{n} \frac{\left(j_{k}(\alpha)\right)^{2 \alpha}}{\left(J_{\alpha}^{\prime}\left(j_{k}(\alpha)\right)\right)^{2}}\left(f\left(\frac{j_{k}(\alpha)}{\tau}\right)+f\left(-\frac{j_{k}(\alpha)}{\tau}\right)\right)\right|<\delta
$$

for all $n \geq N_{\delta}$. Setting

$$
S_{n}:=\sum_{k=N_{\delta}}^{n} \frac{\left(j_{k}(\alpha)\right)^{2 \alpha}}{\left(J_{\alpha}^{\prime}\left(j_{k}(\alpha)\right)\right)^{2}}\left(f\left(\frac{j_{k}(\alpha)}{\tau}\right)+f\left(-\frac{j_{k}(\alpha)}{\tau}\right)\right),
$$

we see that for $n>N_{\delta}$ we have

$$
\begin{aligned}
\left|\sum_{k=N_{\delta}}^{n} \frac{\left(j_{k}(\alpha)\right)^{2 \alpha}}{\left(J_{\alpha}^{\prime}\left(j_{k}(\alpha)\right)\right)^{2}} \psi_{\varepsilon}\left(\frac{j_{k}(\alpha)}{\tau}\right)\right| \\
=\left|\sum_{k=N_{\delta}}^{n} \frac{\left(j_{k}(\alpha)\right)^{2 \alpha}}{\left(J_{\alpha}^{\prime}\left(j_{k}(\alpha)\right)\right)^{2}} \varphi_{\varepsilon}\left(\frac{j_{k}(\alpha)}{\tau}\right)\left(f\left(\frac{j_{k}(\alpha)}{\tau}\right)+f\left(-\frac{j_{k}(\alpha)}{\tau}\right)\right)\right| \\
=\left|\varphi_{\varepsilon}\left(\frac{j_{n}(\alpha)}{\tau}\right) \cdot S_{n}+\sum_{k=N_{\delta}}^{n-1}\left(\varphi_{\varepsilon}\left(\frac{j_{k}(\alpha)}{\tau}\right)-\varphi_{\varepsilon}\left(\frac{j_{k+1}(\alpha)}{\tau}\right)\right) \cdot S_{k}\right| \\
<\left(\left|\varphi_{\varepsilon}\left(\frac{j_{n}(\alpha)}{\tau}\right)\right|+\sum_{k=N_{\delta}}^{\infty}\left|\varphi_{\varepsilon}\left(\frac{j_{k}(\alpha)}{\tau}\right)-\varphi_{\varepsilon}\left(\frac{j_{k+1}(\alpha)}{\tau}\right)\right|\right) \cdot \delta \\
<(1+\pi) \delta
\end{aligned}
$$

by Lemma 2 . Therefore,

$$
\left|\sum_{k=N_{\delta}}^{\infty} \frac{\left(j_{k}(\alpha)\right)^{2 \alpha}}{\left(J_{\alpha}^{\prime}\left(j_{k}(\alpha)\right)\right)^{2}} \psi_{\varepsilon}\left(\frac{j_{k}(\alpha)}{\tau}\right)\right|<(1+\pi) \delta .
$$

The remainder of the proof is analogous to that of Theorem 1.

Proof of Theorem 4. If $f$ satisfies the conditions of Theorem 4, then applying Theorem 1 to the function $f(z) \overline{f(-\bar{z})}$, we get

$$
\begin{aligned}
\int_{0}^{\infty} x^{2 \alpha+1} & \Re e(f(x) \overline{f(-x)}) d x \\
= & \frac{2}{\tau^{2 \alpha+2}} \sum_{k=1}^{\infty} \frac{\left(j_{k}(\alpha)\right)^{2 \alpha}}{\left(J_{\alpha}^{\prime}\left(j_{k}(\alpha)\right)\right)^{2}} \mathfrak{R e}\left(f\left(\frac{j_{k}(\alpha)}{\tau}\right) \overline{f\left(-\frac{j_{k}(\alpha)}{\tau}\right)}\right),
\end{aligned}
$$

where the series on the right-hand side of (18) is absolutely convergent. 
Again, formula (2) applied to the function $(f(z)+f(-z))(\overline{f(\bar{z})+f(-\bar{z})})$ gives

$$
\begin{aligned}
& \int_{0}^{\infty} x^{2 \alpha+1}|f(x)+f(-x)|^{2} d x \\
& =\frac{2}{\tau^{2 \alpha+2}} \sum_{k=1}^{\infty} \frac{\left(j_{k}(\alpha)\right)^{2 \alpha}}{\left(J_{\alpha}^{\prime}\left(j_{k}(\alpha)\right)\right)^{2}}\left|f\left(\frac{j_{k}(\alpha)}{\tau}\right)+f\left(-\frac{j_{k}(\alpha)}{\tau}\right)\right|^{2}
\end{aligned}
$$

Now writing $|f(x)+f(-x)|^{2}$ as $|f(x)|^{2}+|f(-x)|^{2}+2 \mathfrak{R} e(f(x) \overline{f(-x)})$ and

$$
\left|f\left(\frac{j_{k}(\alpha)}{\tau}\right)+f\left(-\frac{j_{k}(\alpha)}{\tau}\right)\right|^{2}
$$

as

$$
\left|f\left(\frac{j_{k}(\alpha)}{\tau}\right)\right|^{2}+\left|f\left(-\frac{j_{k}(\alpha)}{\tau}\right)\right|^{2}+2 \mathfrak{R} e\left(f\left(\frac{j_{k}(\alpha)}{\tau}\right) \overline{f\left(-\frac{j_{k}(\alpha)}{\tau}\right)}\right)
$$

and using (18), we obtain

$$
\begin{aligned}
\int_{0}^{\infty} x^{2 \alpha+1} & \left(|f(x)|^{2}+|f(-x)|^{2}\right) d x \\
& =\frac{2}{\tau^{2 \alpha+2}} \sum_{k=1}^{\infty} \frac{\left(j_{k}(\alpha)\right)^{2 \alpha}}{\left(J_{\alpha}^{\prime}\left(j_{k}(\alpha)\right)\right)^{2}}\left(\left|f\left(\frac{j_{k}(\alpha)}{\tau}\right)\right|^{2}+\left|f\left(-\frac{j_{k}(\alpha)}{\tau}\right)\right|^{2}\right),
\end{aligned}
$$

which is equivalent to $(5)$.

\section{BIBLIOGRAPHY}

1. R. P. Boas, Jr., Inequalities between series and integrals involving entire functions, J. Indian Math. Soc. 16 (1952), 127-135.

2. __ Entire functions, Academic Press, New York, 1954.

3. __ Summation formulas and band-limited signals, Tôhoku Math. J. 24 (1972), 121-125.

4. C. Frappier and P. Olivier, A quadrature formula involving zeros of Bessel functions, Math. Comp. 60 (1993), 303-316.

5. C. Frappier and Q. I. Rahman, Une formule de quadrature pour les fonctions entières de type exponentiel, Ann. Sci. Math. Québec 10 (1986), 17-26.

6. A. R. Harvey, The mean of a function of exponential type, Amer. J. Math. 70 (1948), 181-202.

7. M. Plancherel and G. Pólya, Fonctions entières et intégrales de Fourier multiples, Comment. Math. Helv. 9 (1937), 224-248; 10 (1938), 110-163.

8. G. N. Watson, $A$ treatise on the theory of Bessel functions, 2nd ed., Cambridge Univ. Press, Cambridge, 1945.

9. E. T. Whittaker and G. N. Watson, A course of modern analysis, 4th ed., Cambridge Univ. Press, Cambridge, 1940.

Département de Mathematiques et de Statistique, Universite de Montréal, MonTREAL, QuEBEC, CANADA H3C 3J7

E-mail address, G. Grozev: grozevg@ere.umontreal.ca

E-mail address, Q. Rahman: rahmanqi@ere.umontreal.ca 